\title{
Assessing muscular fatigue with a portable tremor measurement system suitable for field use
}

\author{
TRACI L. GALINSKY and ROGER R. ROSA \\ National Institute for Occupational Safety and Health, Cincinnati, Ohio \\ and University of Cincinnati, Cincinnati, Ohio \\ and \\ DANIEL D. WHEELER \\ University of Cincinnati, Cincinnati, Ohio
}

\begin{abstract}
The utility of a portable tremor measurement system for detecting muscle fatigue was evaluated. Static arm extension was used to induce fatigue. A probe containing two accelerometers, held in the extended hand, recorded horizontal and vertical tremor oscillations in the 1-to $18-\mathrm{Hz}$ range. Several summary amplitude and frequency measures were then derived to analyze hand/arm tremor in the unfatigued and the fatigued states. Large increases from unfatigued to fatigued states were observed in all of the amplitude measures in both axes. Summary frequency measures were far less sensitive to fatigue. Correlations between consecutively measured observations revealed high levels of reliability $(r>.80)$ in all of the amplitude measures, but not in the frequency measures. The most robust index of fatigue was the total power in the entire 1to $18-\mathrm{Hz}$ spectrum. The other amplitude measures, however, are suitable for examining the effects of fatigue in isolated portions of the spectrum.
\end{abstract}

Increased recognition of the human cost of work-related musculoskeletal trauma has expanded research into the risk factors contributing to such trauma (Armstrong, Fine, Goldstein, Lifshitz, \& Silverstein, 1987; Putz-Anderson, 1988; Silverstein, Fine, \& Armstrong, 1986). Taskrelated factors such as awkward postures, excessive repetition, or high degrees of force, as well as job-related factors such as long hours of work and inadequate rest breaks, are presumed to increase overexertion and thus increase the risk of trauma. Proper evaluation of these factors requires an accurate and reliable characterization of the exertion or fatigue produced by them. Jobs could then be redesigned to minimize such fatigue.

One behavioral manifestation of fatigue appears in the form of muscular tremor. Whenever a body part is to be moved or maintained in position, the muscular effort required is inevitably accompanied by involuntary oscillations in the muscle(s) involved (Freund, 1983; Marsden, 1978). These oscillations, termed physiological tremor, are the normal consequence of voluntary muscle contraction and are not viewed as a symptom of fatigue. Physiological tremor consists of arhythmic movements occurring at frequencies predominantly in the range of 7-12 $\mathrm{Hz}$. These movements are of such low amplitude that they are

Correspondence should be addressed to Traci L. Galinsky, National Institute for Occupational Safety and Health, Division of Biomedical and Behavioral Science, Robert A. Taft Laboratories, 4676 Columbia Parkway, Cincinnati, OH 45226-1998. not visible to the naked eye. The onset of fatigue, however, produces enhanced physiological tremor, characterized by large amplitude oscillations that are regular or monorhythmic, with an enhanced peak frequency of between 7 and $12 \mathrm{~Hz}$ (Freund, Hefter, Homberg, \& Reiners, 1984; Young, 1984).

Normal and enhanced physiological tremors appear to be governed by different mechanisms. Normal tremor has been attributed to factors such as mechanical resonance (Rietz \& Stiles, 1974), the filter properties of muscle (Marshall \& Walsh, 1956), and the inherent characteristics of motor unit activity (Marsden, 1978). Evidence suggests that enhanced physiological tremor is regulated by oscillation in the stretch reflex arc (Young \& Hagbarth, 1980 ), whose rate is controlled by the combined influence of the onset firing rate of motor units and the filtering effects of muscle (Freund et al., 1984).

It has long been recognized that muscle fatigue raises the amplitude of physiological tremor. Indeed, Leon Binet's (1920) second law of tremor holds that "tremor increases as the result of muscular contraction and becomes exaggerated under the influence of work" (p. 104). The sensitivity of enhanced physiological tremor as a fatigue indicator is revealed in studies demonstrating that strong muscular contractions lasting only $5 \mathrm{~min}$ result in enhanced tremor lasting for several hours (Furness \& Jessop, 1976; Furness, Jessop, \& Lippold, 1977).

Most of the research on normal and enhanced tremor has focused on exploring underlying mechanisms. This research has traditionally made use of bulky, nonportable equipment for electromyographic and accelerometric 
recording and subsequent spectral analysis in the laboratory (see, e.g., Allum \& Hulliger, 1982; Brown, Rack, \& Ross, 1982; Brumlik, 1962; Elble \& Randall, 1978; Halliday \& Redfearn, 1956; Lippold, 1970; Matthews \& Muir, 1980; Rietz \& Stiles, 1974). These procedures are arduous and time-consuming, requiring immobilization of the subject, careful isolation of muscles or muscle groups, electrode hookup, and so forth. As such, these procedures are not practical for measuring tremor as an index of fatigue in the workplace. Such an application requires a portable apparatus, as well as procedures that can be easily administered in a timely fashion.

Previous investigators have made use of a portable hand stylus device to examine muscular tremor (Borg, 1969; Borg \& Sjöberg, 1981; Gutin, Fogel, Meyer, \& Jaeger, 1974; Rosa \& Colligan, 1988; Rosa, Colligan, \& Lewis, 1989; Rosa, Wheeler, Warm, \& Colligan, 1985). In these experiments, hand tremor was studied by having subjects attempt to center a conductive metal wand inside a small opening sensitive to contact. Increased fatigue was indexed by more frequent contact of the wand with the sides of the opening. This technique is limited, however, because it assesses hand tremor only in terms of the discrete events that occur when the wand touches the side of the opening. This measure provides no information about what $\alpha$ curs between the events. Furthermore, the magnitude of the event obtained with this method is determined to a large extent by the size of the opening.

Our aim was to develop a portable apparatus that would be easy to use and that would provide reliable measures of tremor oscillations over a wide amplitude range. A microcomputer-based prototype of such a system has now been developed, in which accelerometers in a probe held by the subject at arm's length transduce the tremor. Fast Fourier transforms provide power spectra of the tremor being measured.

The objective of the present study was to demonstrate the usefulness of a system for detecting muscular fatigue and to determine which summary measures of tremor should be reported by the system. This evaluation involved deciding which of the various measures produced by the system would be most sensitive to fatigue, and determining which measures would be most reliable.

Static exertion in muscles of the hand, arm, and shoulder was used to induce fatigue. Our operational definition of fatigue consisted of the duration of exertion required to produce a level of discomfort judged to be unacceptable by the subject. Thus, subjects were matched on a perceptual level in terms of the amount of work they were willing to perform. Our criterion is analogous to the "maximal voluntary contraction" standard used in studies of muscular exertion (see Kroemer \& Marras, 1981). It is also consistent with Bartley (1965), who noted that work fatigue can be considered as a perceptual problem involving "an experienced self-evaluation" that encompasses motivational and sensory factors (p. 7).

\section{METHOD}

\section{Subjects}

Eighty individuals ( 35 women and 45 men) with no physical impairments in the hands, arms, or shoulders served as subjects. Their ages ranged from 18 to 44 , with a mean age of 20.3. All subjects were recruited from the University of Cincinnati Psychology Department subject pool and received course credit for their participation.

\section{Apparatus}

Accelerometry assembly. Two Wilcoxon Research piezoelectric accelerometers (Model WR-137), having a sensitivity of $85 \mathrm{mV}$ per gauss and a frequency response of 1-830 Hz, were mounted at right angles inside a handgrip unit (the tremor probe) constructed from a T-shaped piece of plastic pipe. They were used to record tremor in the horizontal and vertical axes. The horizontal portion of the $T$ was $8 \mathrm{~cm}$ long, with an inside diameter of $4 \mathrm{~cm}$. The hollow tube was used by the subject as a target sight in order to establish and maintain the correct orientation. The vertical handgrip portion of the $T$ was $9 \mathrm{~cm}$ long, with an outside diameter of $3.9 \mathrm{~cm}$. The entire unit weighed $76 \mathrm{~g}$, which is less than $3 \%$ of the weight of a petite woman's arm (approximately $2 \mathrm{~kg}$ ).

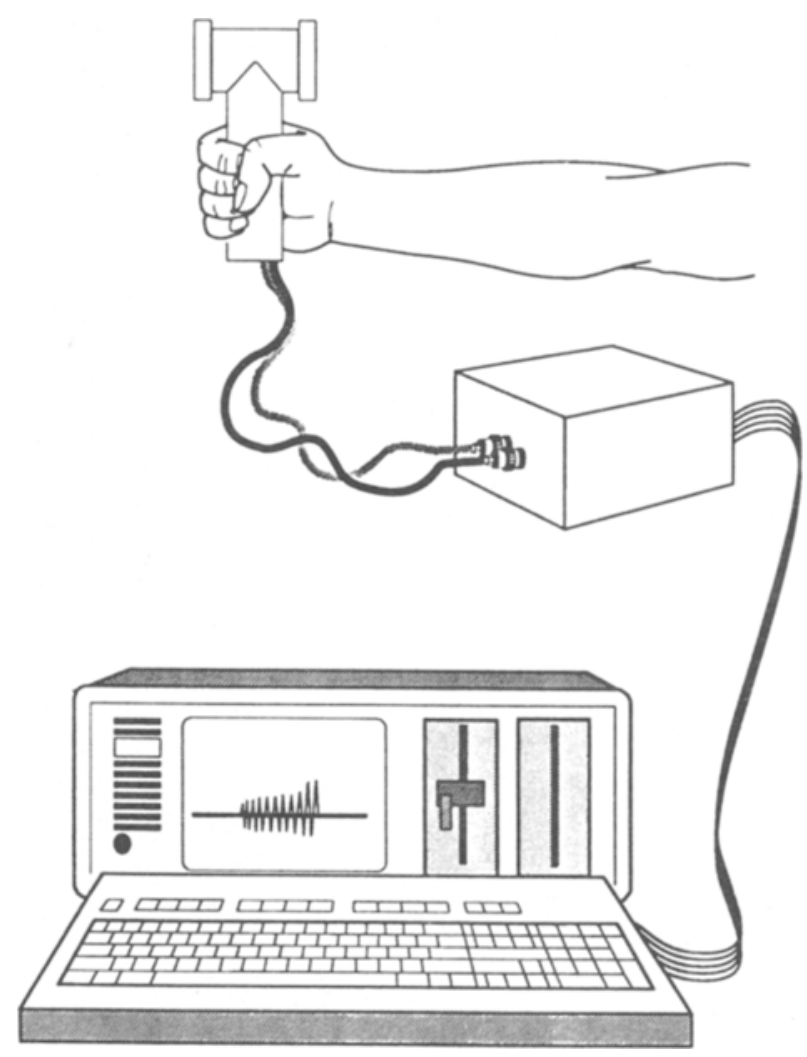

Figure 1. The tremor measurement system. 
Amplifier interface and A/D converter. Signals generated by the accelerometers were amplified with a MetraByte four-channel Simultaneous Sample and Hold Module (Model SSH-4) containing built-in amplifiers. A metal enclosure shielded this module from noise. Analog-todigital conversion was executed with a MetraByte DAS20 Analog and Digital Interface Board mounted in a Compaq-Plus portable microcomputer (MS-DOS operating system). An illustration of the entire apparatus, including the tremor probe, is presented in Figure 1.

Two channels of the SSH-4 Module were used. The dccoupled input to the amplifier was matched to the highimpedance ac signal of the accelerometers with a highpass filter. The low-frequency cutoff of the filter was $.1 \mathrm{~Hz}$. The switches on the amplifiers were set for a gain of 100 . Pilot testing indicated that this setting provided adequate sensitivity for measuring low levels of tremor and sufficient dynamic range for high levels of tremor.

Sampling characteristics. A sample rate of $60 \mathrm{~Hz}$ was selected so that power line noise would alias to a frequency of zero and be eliminated from the spectrum. The sample rate allowed detection of signals of up to $30 \mathrm{~Hz}$, which encompasses the range of physiological tremor.

Fast Fourier transform. The optimum sample size for a fast Fourier transform is a power of two. A sample size of 512 was selected to be as large as possible while still allowing rapid completion of the fast Fourier transform. At $60 \mathrm{~Hz}$, each sample of 512 requires $8.53 \mathrm{sec}$.

\section{Procedure}

After recording the relevant demographic data and obtaining informed consent, each subject was seated in a straight-backed chair next to a table supporting the tremorrecording apparatus.

Before the actual measurement session, each participant was instructed to hold the tremor probe in the dominant hand, and to fully extend the arm, pointing the probe directly at a vertical column of circles drawn on the wall $4 \mathrm{ft}$ away. They were asked to sight with one eye through the opening in the probe and focus on a circle that would keep the arm at a right angle to the body. A patch was placed over the other eye to prevent diplopia during sighting. The subject was then allowed to rest while the remaining instructions were given.

For the actual measurement, an upper limit of $10 \mathrm{~min}$ of arm extension was selected. This limit was based on previous research (reviewed by Chaffin, 1973), which indicated that sustained static exertions typically lead to sensations of discomfort or pain within a few minutes. During measurement, the subject was positioned in an upright posture with the back flush to the chair. The subject then pointed the tremor probe at the circle, and the experimenter initiated the measurement session on the computer. Each subject was instructed to maintain position, keeping the arm and hand as still and straight as possible, while gripping the tremor probe firmly but comfortably, for as long as possible up to the 10 -min limit. The subject was asked to inform the experimenter, before lowering the arm, that he or she was ready to quit if an unacceptable level of discomfort was reached before the 10-min limit. The experimenter then terminated the measuring process on the computer, and the subject lowered the arm.

Two consecutive measurements were taken during each minute of the session. The first measurement commenced $10 \mathrm{sec}$ after the start of arm extension, and the second measurement began $20 \mathrm{sec}$ after the start of the arm extension. Thirty seconds into the session, the first two measures were automatically stored on disk. The same sequence commenced $10 \mathrm{sec}$ into each of the remaining minutes. The duration of arm extension ranged from 2 to $9 \mathrm{~min}$, with a mode of $4 \mathrm{~min}$, a mean of $5 \mathrm{~min}$, and a standard deviation of $2 \mathrm{~min}$. This variability in endurance was not surprising, given the heterogeneity of our subject sample in terms of age, physique, and so forth.

\section{Tremor Summary Measures}

For each sample, the fast Fourier transform produced a power spectrum of 256 discrete points covering a frequency range of $0-30 \mathrm{~Hz}$. The first step in data reduction was to partition the values in groups of 8 summed points, thus producing a spectrum of 32 points. Each point represented the power in a frequency band of $.94 \mathrm{~Hz}$ $(30 / 32)$. (Subsequent discussion will treat these as though they were $1-\mathrm{Hz}$ bands.) The bottom band and the top 13 bands were dropped because they represented frequencies outside the range of interest in this study. Each point in the remaining spectrum represented an approximate 1$\mathrm{Hz}$ band within a total range of approximately $1-18 \mathrm{~Hz}$ $(.94-17.70 \mathrm{~Hz})$.

Amplitude measures. Amplitude measures included the total power at each frequency from $1-18 \mathrm{~Hz}$, the total power within each third of this frequency range (1-6, $7-12$, and $13-18 \mathrm{~Hz}$ ), and the total power summed over the entire frequency range $(1-18 \mathrm{~Hz})$.

The power measures were transformed to natural logarithms. In the case of the summary amplitude measures (sum of the power at 1-6, 7-12, 13-18, and $1-18 \mathrm{~Hz}$ ), the raw power values from each frequency contained in the measure were summed, and the summed values were transformed.

Frequency measures. Two types of measures were used to describe tremor frequency. Peak frequency measures specified the frequency at which most of the power was exhibited. Median frequency measures designated the frequency that separated the spectrum (or a portion of the spectrum) into halves of equal power. Two peak frequency measures were used, including the maximum amplitude frequency within the entire 1- to $18-\mathrm{Hz}$ range (Max 1-18) and the maximum amplitude frequency within the 7- to $12-\mathrm{Hz}$ physiological tremor range (Max $7-12)$. These measures were based on the 18-point power spectra. Two median frequency measures were also calculated from portions of the original 256-point spectra, including: the frequency below which $50 \%$ of the energy in the 1 - to $18-\mathrm{Hz}$ portion of the spectrum was contained (Med1-18), 
and the frequency that divided the $7-$ to $12-\mathrm{Hz}$ band into equal-energy halves (Med7-12). Other researchers have used similar descriptors of central tendency in tremor power spectra (Chapman et al., 1990).

\section{Statistical Analysis}

We were primarily interested in determining which aspects of tremor, as measured by the prototype system, were most closely associated with fatigue. Consequently, we used each of the measures described above to compare tremor observed in an unfatigued state with that observed in a fatigued state. Fatigue, in our case, was the point at which the subjects were unwilling or unable to maintain arm extension.

Repeated measures analyses of variance (ANOVAs) were computed separately for each axis (horizontal and vertical) to compare differences between the unfatigued and fatigued states. A separate ANOVA was computed for each amplitude and frequency measure. For each measure, values recorded immediately after the first minute of arm extension (i.e., within 70-90 sec) were compared with the corresponding values recorded from the last minute of arm extension (unfatigued and fatigued states, respectively). (Tremor records obtained before 1 full minute of extension were discarded because of artifactual movements used by the subjects to adjust the arm at the start of the session.) These ANOVAs were computed on the entire sample of 80 subjects.

To examine minute-to-minute changes in tremor amplitude, additional repeated measures ANOVAs were computed on a subsample of 17 subjects who reached the modal duration of $4 \mathrm{~min}$ of arm extension. For each axis, a separate 4 (minutes) $\times 17$ (subjects) ANOVA was computed for each frequency $(1-18 \mathrm{~Hz})$, using the total power at that frequency as the dependent variable.

For brevity, the $F$ values reported in the Results section read "greater than or equal to," indicating that all of the $F$ tests for a particular set of analyses exceeded that value.

Effect size. To compare each measure as a fatigue indicator relative to that of the other measures, it was necessary to standardize the differences between the values obtained in fatigued and unfatigued states. For each measure, effect size was computed as the difference between the mean values obtained in unfatigued and fatigued states, divided by the pooled standard deviation. Thus, the effect size for a given measure represents the number of standard deviations between the unfatigued and fatigued states. This method of standardizing effects is common in meta-analyses (Hedges \& Olkin, 1985).

Proportion of accountable variance. Another way to compare the various measures was to calculate the proportion of variance associated with fatigue. These values were estimated for each dependent variable from the analyses of variance $(N=80)$, using the formula given in Hays $(1981$, p. 365$)$.
Reliability correlations. The utility of any measurement instrument remains open to question until its reliability is demonstrated. We estimated the reliability (stability) of the system by computing Pearson product-moment correlations between the two consecutively measured observations recorded after the $1 \mathrm{st}$ minute of arm extension (unfatigued state), and between the two consecutively measured observations recorded during the last minute of arm extension (fatigued state). This procedure is analogous to the test-retest reliability procedure described by Guilford (1954).

\section{RESULTS}

\section{Sensitivity to Fatigue}

Individual frequencies (1-18 Hz). Mean power in unfatigued and fatigued states is shown for each frequency in Figure 2. (Data are from the entire sample of $80 \mathrm{sub}$ jects.) In both axes, and for each frequency, the amplitude of tremor in the fatigued state is significantly larger than the amplitude of tremor seen in the unfatigued state $[F(1,79) \geq 42.86, p<.0001]$. (Note: All $F$ s exceeded this value.) Within each axis, the spectra for fatigued and unfatigued states are fairly similar in shape. Between each axis, however, the shapes of the spectra are notably different. Tremor in both axes yielded two peaks-one at approximately $2-3 \mathrm{~Hz}$ and the other in the $7-$ to $11-\mathrm{Hz}$ range. The lower frequency peak, however, is more pronounced in the vertical axis, and the higher frequency peak is more pronounced in the horizontal axis.

Minute-to-minute changes in tremor amplitude are shown for each frequency in Figure 3. (These data are from 17 subjects who reached $4 \mathrm{~min}$ of arm extension.) As shown, gradual increases in tremor amplitude with each minute of arm extension are apparent in both axes. These impressions are supported by a significant effect for time at each frequency in both axes $[F(3,48) \geq 3.88$, $p \leq .014]$, with the exception of $12 \mathrm{~Hz}$ in the vertical axis $[F(3,48)=2.17, p=.10]$.

The shapes of the spectra in Figures 2 and 3 are essentially identical, with two visible peaks differing in their relative magnitudes for the two axes. The peaks between 7 and $11 \mathrm{~Hz}$ are particularly evident for the horizontal axis, becoming more pronounced as fatigue accrues. These power spectra typify "enhanced physiological tremor"' (Young, 1984).

The 2- to $3-\mathrm{Hz}$ peaks can be attributed to two possible sources. One of these sources is mechanical resonance. Passive mass-spring systems, such as human limbs, oscillate at a resonant frequency that is inversely related to mass. The resonant frequency of the human arm is approximately 2- to 3-Hz (Joyce \& Rack, 1974), and previous studies have revealed 3-Hz peaks in human arm tremor (Fox \& Randall, 1970; Robson, 1962). Thus, mechanical resonance could contribute to the $2-$ to $3-\mathrm{Hz}$ peaks observed in the present study. Voluntary adjust- 


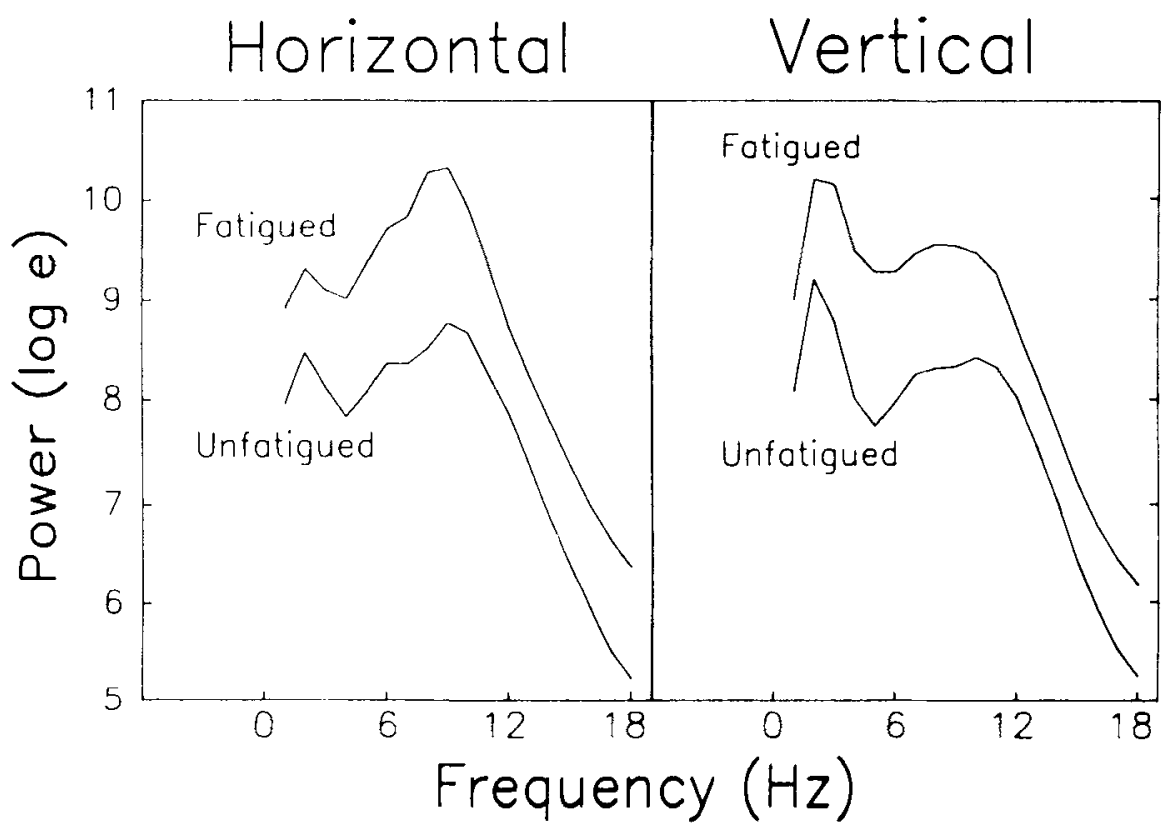

Figure 2. Tremor power spectra in the horizontal and vertical axes in unfatigued and fatigued states. These values are based on the entire sample of 80 subjects.

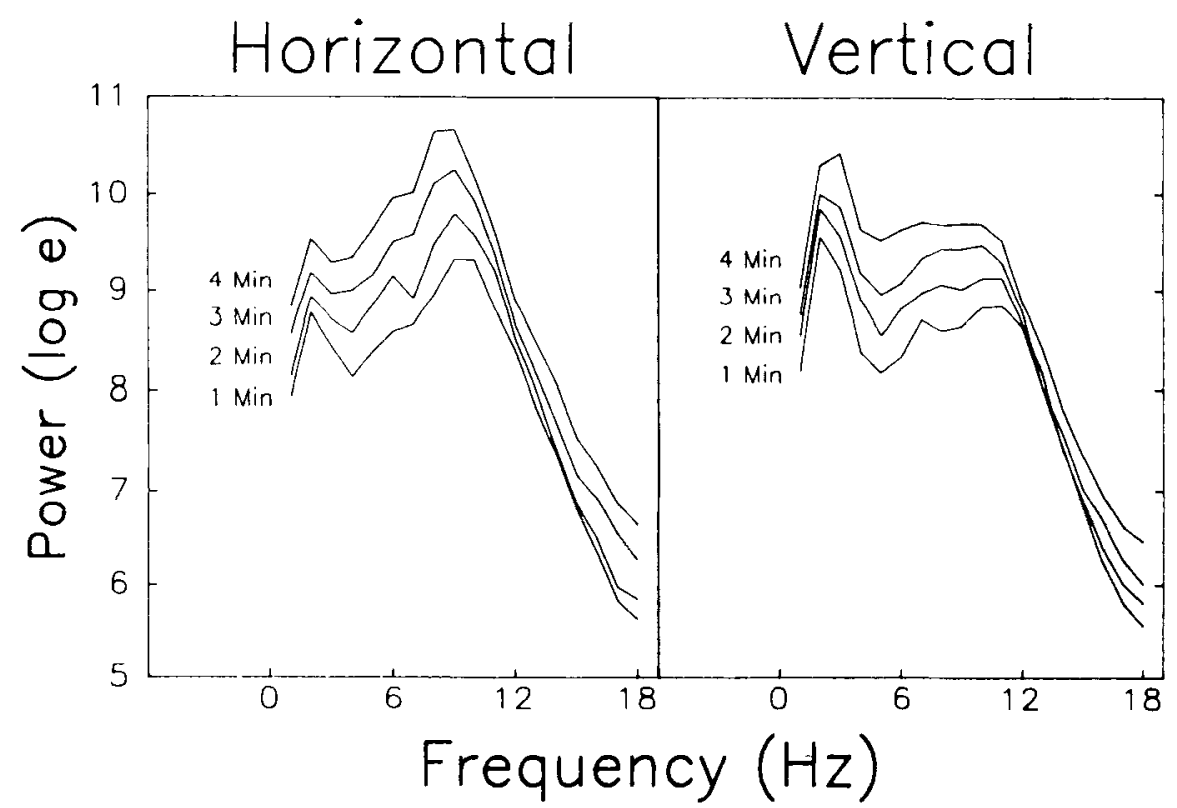

Figure 3. Minute-to-minute tremor power spectra in the horizontal and vertical axes. These values are based on 17 subjects who maintained arm extension for 4 min. 


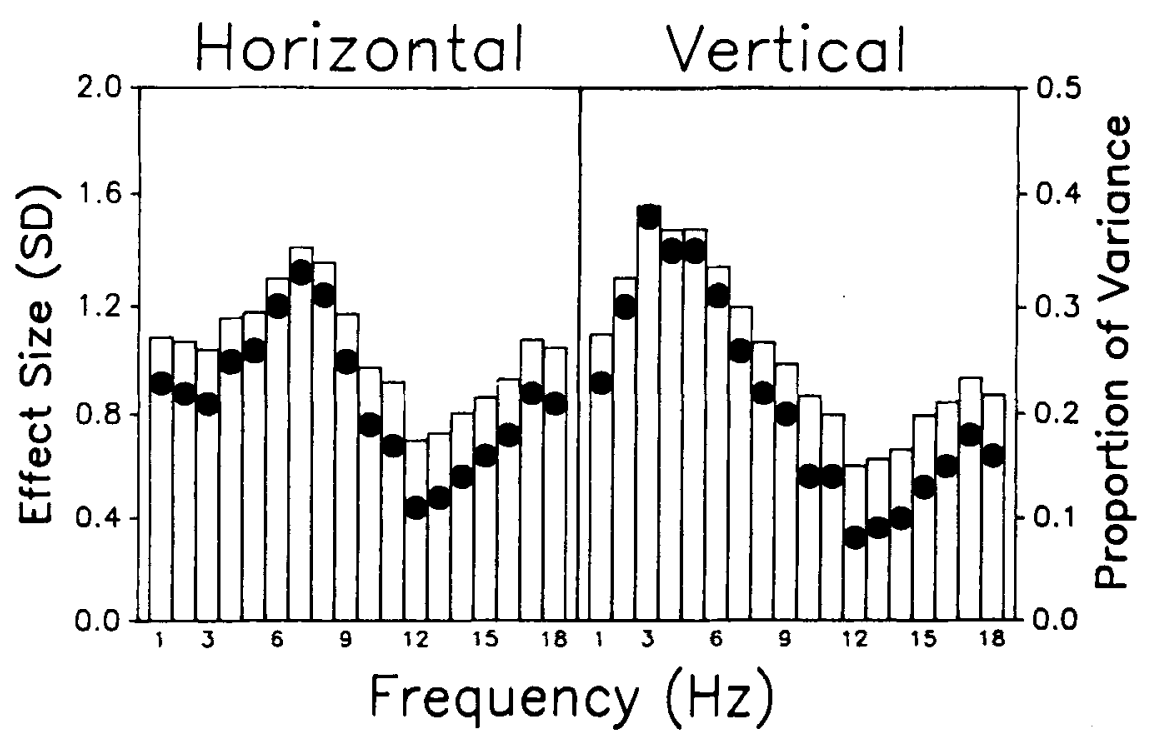

Figure 4. Effect sizes for fatigue, and proportion of variance associated with fatigue, at each frequency in the horizontal and vertical axes. Effect sizes are indicated by bars, and proportions of variance are indicated by circles.

ments to keep the tremor probe aligned with the visual target, however, could also produce peak frequencies in this range. The higher peak in the vertical axis may be attributable to the effects of gravity, and/or differences in limb kinematics in the horizontal and vertical planes.

The effect sizes for fatigue at each frequency are presented in Figure 4, accompanied by the corresponding values for the proportion of variance associated with fatigue. (Values are from the entire sample of 80 subjects.) As shown in this figure, the largest effects of fatigue occurred at different frequencies for the two axes. In the horizontal axis, fatigue-induced increments in tremor amplitude were largest in the 6- to $9-\mathrm{Hz}$ range. In the vertical axis, these increments were largest in the 2- to 6- $\mathrm{Hz}$ range.

Summary amplitude measures. Mean tremor amplitude in various portions of the $1-$ to $18-\mathrm{Hz}$ spectrum in the unfatigued and fatigued states are depicted for each axis in the top half of Figure 5. The values obtained for these measures are strikingly similar for the two axes. In each case, tremor amplitude at $1-6$ and $7-12 \mathrm{~Hz}$, as well as in the entire 1- to $18-\mathrm{Hz}$ spectrum, is uniformly higher than that at 13-18 Hz. Moreover, fatigue produced comparable increases in mean tremor amplitude in all portions of the spectrum in the two axes. The effect of fatigue was significant for each measure in both axes $[F(1,79) \geq$ $91.05, p<.0001]$

The relative extent of fatigue's influence on the above measures, in terms of effect sizes and proportion of accountable variance, is shown in the left half of Figure 6. It is apparent from Figure 6 that the largest effect of fatigue occurred in the lower two frequency bands (1-6 and $7-12 \mathrm{~Hz}$ ) and in the entire 1- to $18-\mathrm{Hz}$ spectrum. Notable differences between the two axes are evident in the two lower ranges. The 1- to 6-Hz band shows the larger effect in the vertical axis compared to the $1-$ to $6-\mathrm{Hz}$ band in the horizontal axis, and the $7-$ to $12-\mathrm{Hz}$ band shows the larger effect in the horizontal axis compared to the $7-$ to $12-\mathrm{Hz}$ band in the vertical axis. Of the four measures, the most stable index of fatigue appears to be the total power in the entire spectrum. Total power shows a sizable fatigue effect that does not vary widely among subjects and is independent of the axis in which tremor oscillations are recorded.

Frequency measures. Mean values for the four measures of tremor frequency in unfatigued and fatigued states are presented in the bottom half of Figure 5. Overall, the peak and median frequency of tremor fell predominantly in the range between 6 and $10 \mathrm{~Hz}$. The exception was Max1-18 in the vertical axis, which clearly revealed the influence of the low frequency peak in the power spectra of that axis (see Figure 2). Although statistically significant in some cases, changes in tremor frequency brought on by fatigue were small (less than $1 \mathrm{~Hz}$ ). In both axes, Max7-12 and Med7-12 decreased significantly from 1 min to the last minute of arm extension $[F(1,79) \geq$ $6.42, p<.01]$. In the vertical axis, Med1-18 decreased significantly from $1 \mathrm{~min}$ to the last minute of arm extension $[F(1,79)=12.09, p<.0008]$. No other significant effects of fatigue were evident in the frequency measures.

The effect sizes for fatigue, along with the proportion of variance associated with fatigue in the four frequency measures, are presented for each axis in the right half of Figure 6. As shown in this figure, the effect size associated with fatigue in the frequency measures was substantially smaller than the effect size in the amplitude measures. Indeed, the proportion of variance associated with fatigue in the frequency measures never exceeded 


\section{Horizontal Vertical}
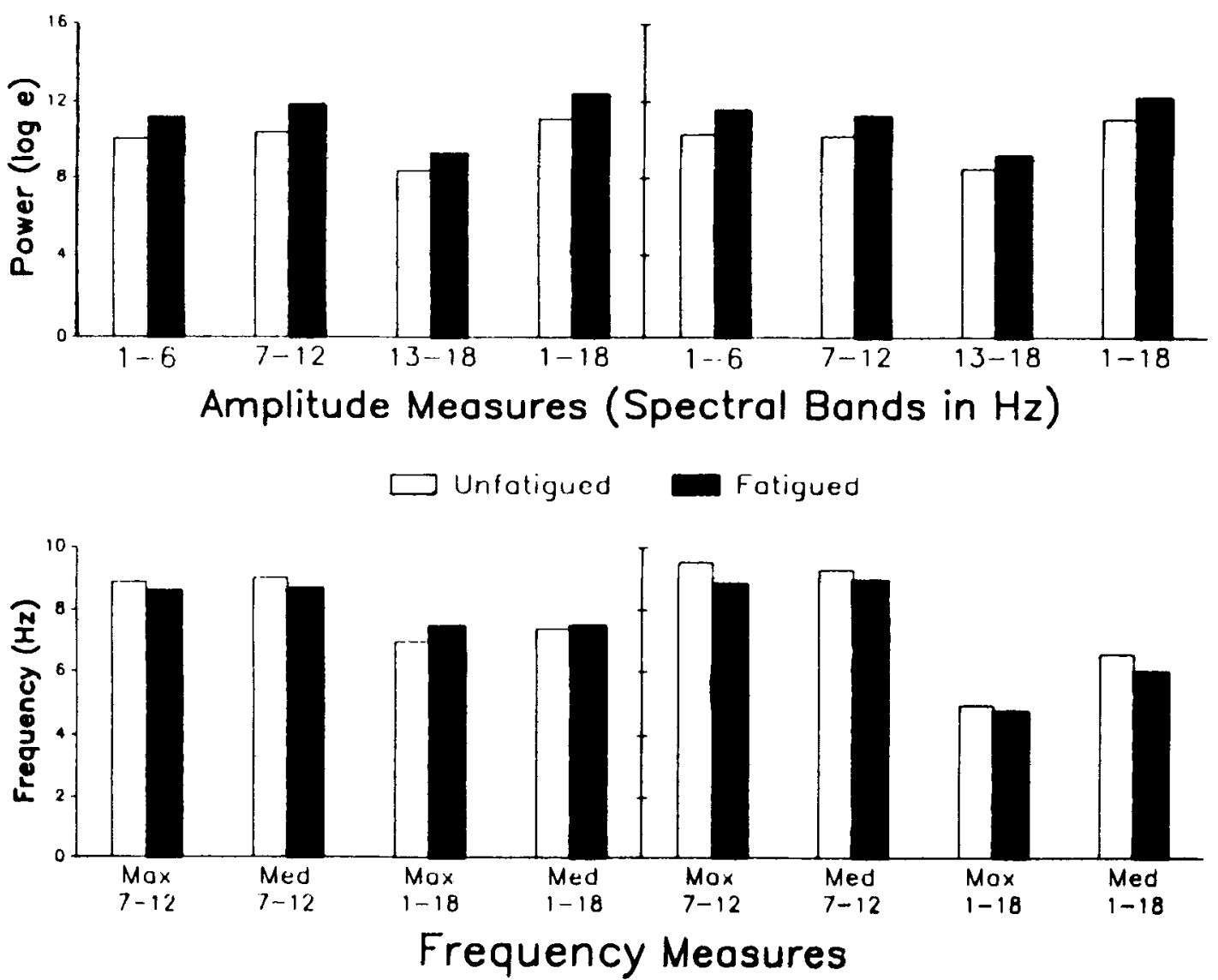

Figure 5. Tremor amplitude and frequency summary measures in the horizontal and vertical axes in unfatigued and fatigued states.

$6 \%$. By contrast, the proportion of variance associated with fatigue in the amplitude measures ranged from $16 \%$ to $39 \%$ (see Figure 6).

\section{Reliability of Measures}

Correlations between consecutively measured observations in the unfatigued and fatigued states are presented in Table 1. All of the correlations in this table are statistically significant $(p \leq .001$ ). Those above .80 (in boldface) were arbitrarily designated to be highly reliable. Of the four amplitude measures, power in the $7-$ to $12-\mathrm{Hz}$ and $13-$ to $18-\mathrm{Hz}$ bands, as well as the power registered in the entire 1- to $18-\mathrm{Hz}$ spectrum, consistently exhibited high levels of reliability in each of the two axes in both unfatigued and fatigued states. For each of the four amplitude measures, these correlations are elevated somewhat in the fatigued compared to the unfatigued state. This effect probably reflects the increase in rhythmicity of tremor oscillations that occurs during enhanced physiological tremor (Young, 1984). Table 1 also shows that Medl-18 is the only frequency measure that demonstrated satisfactory levels of reliability.

\section{DISCUSSION}

\section{Sensitivity to Muscular Fatigue}

Muscle fatigue results in an increase in the amplitude of physiological tremor oscillations (cf. Young, 1984). Several ways of describing this effect using the prototype system were explored.

Large increments in tremor acceleration, produced by extended periods of isotonic contractions, were revealed by all of the system's amplitude measures. Power spectra in the range of 1-18 $\mathrm{Hz}$ showed fatigue-induced increases in power at all frequencies in both the horizontal and vertical planes, with the extent of the increase at specific frequencies differing in the two axes. Thus, although fatigue can be detected at any frequency in either axis with the prototype system, the user may prefer to inspect the changes in amplitude at only those frequencies that illustrate fatigue most dramatically.

Summary amplitude measures also were provided. These measures were used to describe the sum of the power in the $1-$ to $6-\mathrm{Hz}$ band, the $7-$ to $12-\mathrm{Hz}$ band, the 13- to $18-\mathrm{Hz}$ band, and in the entire $1-$ to $18-\mathrm{Hz}$ spec- 


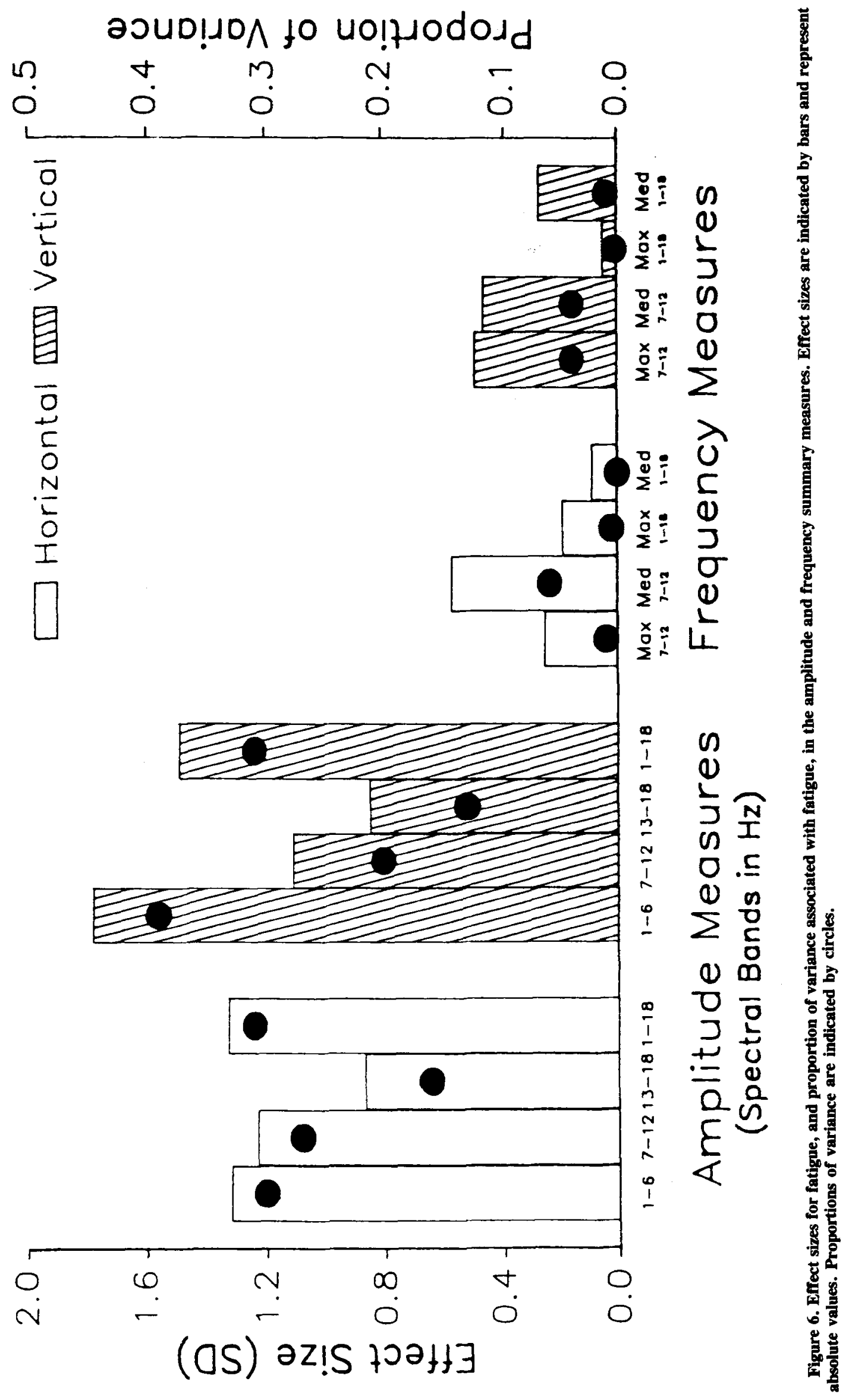


Table 1

Pearson Correlations between Consecutively Measured Observations for the Amplitude and Frequency Summary Measures for Unfatigued and Fatigued States

\begin{tabular}{cccccc} 
& \multicolumn{2}{c}{ Horizontal } & & \multicolumn{2}{c}{ Vertical } \\
\cline { 2 - 3 } \cline { 5 - 6 } Measure & Unfatigued & Fatigued & & Unfatigued & Fatigued \\
\hline Amplitude & & & & & \\
$1-6 \mathrm{~Hz}$ & .73 & .86 & & .67 & .86 \\
$7-12 \mathrm{~Hz}$ & .95 & .95 & & .93 & .96 \\
$13-18 \mathrm{~Hz}$ & .89 & .94 & & .91 & .93 \\
$1-18 \mathrm{~Hz}$ & .89 & .94 & & .85 & .92 \\
Frequency & & & & \\
Max7-12 & .53 & .55 & .56 & .36 \\
Med7-12 & .76 & .66 & .78 & .67 \\
Max1-18 & .59 & .55 & .61 & .66 \\
Med1-18 & .81 & .85 & .86 & .81 \\
\hline
\end{tabular}

Note-Correlations above .80 are in boldface.

trum. Standardizing the size of the fatigue effect among the summary amplitude measures revealed that all of them were sensitive to the effects of fatigue. The size of the effect, however, was dependent on the measure used and the axis recorded. If the sole purpose of using the system is to detect the presence of fatigue, total power in the spectrum would be a suitable measure. This variable appears to be less susceptible to intersubject variability than the other three amplitude measures, and it shows similar increases in the two axes. In addition, the magnitude of the fatigue effect on total power is substantial, accounting for a large proportion of variance. The other three amplitude measures, however, are certainly adequate for examining enhanced tremor in isolated segments of the spectrum.

The measures of peak and median frequency proved to be considerably less sensitive to fatigue. Although fatigue produced statistically significant decreases in some of the frequency measures used in this study, the actual changes were small (less than $1 \mathrm{~Hz}$ ). Inspection of the standardized effect sizes and proportion of variance associated with fatigue (Figure 5) confirms that the frequency measures were much less responsive to fatigue than the amplitude measures. These data are supported by the results of earlier studies, which, taken together, show that the effects of fatigue on peak tremor frequency are inconsistent (Bousfield, 1932; Brooke, Wilson, Dalton, \& Rosenrot, 1985; Eshner, 1897; Fernet, 1872; Iaizzo \& Pozos, 1982; Lippold, Redfearn, \& Vuco, 1960; Stiles, 1976; Stiles \& Rietz, 1977). The variability of the fatigue effect on tremor frequency is demonstrated further in the reliability data of the present study.

\section{Reliability Assessment}

Correlations between consecutively measured observations indicated high levels of reliability $(r>.80)$ for all of the amplitude measures. The only frequency measure with similarly high levels of reliability was Med1-18.

\section{Applications}

The amplitude measures used in this study were more sensitive to fatigue, and more reliable, than the frequency measures used. These results indicate that muscular fatigue in the hand and arm is best described by changes in tremor amplitude as opposed to tremor frequency. This conclusion is consistent with those of Brooke et al. (1985). In view of the finding that amplitude alone is sufficient for describing fatigue, Brooke et al. suggested avoiding the complexities of tremor recordings by simply detecting errors in hand steadiness using the hand stylus technique. While this approach may be adequate in some situations, it is only useful for detecting changes in the largest amplitude components of tremor. These components cannot be expressed with any precision because of the amplitude ceiling imposed by the contact-sensitive opening. The tremor measurement system described in the present report can be used just as easily as handsteadiness measures, and it allows for a graded assessment of changes across a wide range of amplitudes. This affords more opportunity for relative comparisons of fatigue produced by variations in job components such as work duration, rate of movement, tool weight, and so forth. Moreover, in situations where fatigue-induced impairment of fine motor control has potentially negative consequences, the ability to detect even modest changes in tremor amplitude may be useful. (Readers interested in displacement-sensing techniques which also allow continuous amplitude measurement should refer to the work of Freund, 1987, and Kondraske, 1986.)

In the present study, the tremor measurement system was shown to be useful for depicting the accumulation of fatigue in the hand, arm, and shoulder during continuous static exertions. Our apparatus, however, can be adapted easily to measure oscillations from different sites, and our procedure can be modified to measure enhanced tremor resulting from dynamic muscular exertion. Measuring from different sites would require other coupling devices for the accelerometers. For example, they could be attached to a ring worn on the finger to measure finger or hand tremor or, at the other extreme, to the subject's belt to measure body sway. In any case, certain considerations should be made when adapting the apparatus to suit various purposes. For example, the ratio of the weight of the coupling device to the weight of the limb being measured should be as small as possible. It is also important to ensure that each accelerometer maintain its appropriate orientation (horizontal or vertical) with respect to gravity. It is not recommended that the system be used for measuring tremor during dynamic movement of the limb, since no provision has been made to partition the acceleration of tremor from the acceleration of voluntary movement. With our system, enhanced tremor associated with dynamic work should be assessed with static measurements in a pre- to postwork design.

\section{Availability}

Hardware for the apparatus is commercially available. The software, as well as a manual containing a complete description of the system, can be obtained from the authors at the National Institute for Occupational Safety and Health. 


\section{REFERENCES}

Allum, J. H. J., \& Hulliger, M. (1982). Presumed reflex responses of human first dorsal interosseus muscle to naturally occurring twitch contractions of physiological tremor. Neuroscience Letters, 28, 309-314.

Armstrong, T. J., Fine, L. J., Goldstein, S. A., Lifshitz, Y. R., \& Silverstein, B. A. (1987). Ergonomics considerations in hand and wrist tendinitis. Journal of Hand Surgery, 12A, 830-837.

BARTLEY, S. H. (1965). Fatigue: Mechanism and management. Springfield, IL: Charles C Thomas.

Binet, L. (1920). The laws of tremor. London Lancet, 198, 265-266.

BORG, G. (1969). Den fysiska anstrdngningens inverkan pa reaktionstid, flicker-fusion och handstadighet (Rapport 0023). Stockholm: Personaladministrativa Rådet.

BORG, G., \& SJöBERG, H. (1981). The variation of hand steadiness with physical stress. Journal of Motor Behavior, 13, 110-116.

Bousfield, W. A. (1932). The influence of fatigue on tremor. Journal of Experimental Psychology, 15, 104-107.

Brooke, J. D., Wilson, B. A., Dalton, J., \& Rosenrot, P. (1985). Prior physical work intensity increases the amplitude but not the frequency of combined tremor of the hand and finger. Journal of $\mathrm{Hu}$ man Ergology, 14, 71-77.

Brown, T. I. H., Rack, P. M. H., \& Ross, H. F. (1982). Different types of tremor in the human thumb. Journal of Physiology, 332, 113-123.

Brumlik, J. (1962). On the nature of normal tremor. Neurology, 12, 159-179.

Chaffin, D. B. (1973). Localized muscle fatigue: Definition and measurement. Journal of Occupational Medicine, 15, 346-354.

Chapman, L. J., Sauter, S. L., Henning, R. A., Levine, L., MatTHEWS, C. G., \& Peters, H. A. (1990). Early finger tremor differences afier exposure to carbon disulfide-based pesticides. Manuscript submitted for publication.

Elble, R. J., \& RANDALl, J. E. (1978). Mechanistic components of normal hand tremor. Electroencephalography \& Clinical Neurophysiology, 44, 72-82.

ESHNER, A. A. (1897). A graphic study of tremor. Joumal of Experimental Medicine, 2, 301-312.

Fernet, C. (1872). Des tremblements. Paris: Asselin.

Fox, J. R., \& RANDALL, J. E. (1970). Relationship between forearm tremor and the biceps electromyogram. Journal of Applied Physiology, 29, 103-108.

FrEUND, H. J. (1983). Motor unit and muscle activity in voluntary motor control. Physiological Reviews, 63, 387-436.

FreUND, H. J. (1987). Central tremors. Electroencephalography \& Clinical Neurophysiology, 66, S34-S35. (Abstract)

Freund, H. J., Hefter, H., HomberG, V., \& Reiners, K. (1984). Determinants of tremor rate. In L. J. Findley \& R. Capildeo (Eds.), Movement disorders: Tremor (pp. 195-204). New York: Oxford University Press.

FuRness, P., \& JessoP, J. (1976). Prolonged changes in physiological tremor, following a brief maximal voluntary contraction of human muscle. Journal of Physiology, 258, 72-73.

FurNess, P., JESSOP, J., \& LiPPOLD, O. C. J. (1977). Long-lasting increases in the tremor of human hand muscles following brief, strong effort. Journal of Physiology, 265, 821-831.

Guilford, J. P. (1954). Psychometric methods. New York: McGraw-Hill.

Gutin, B., Fogel, K., Meyer, J., \& JAEger, M. (1974). Steadiness as a function of prior exercise. Journal of Motor Behavior, 6, 69-76.

Halliday, A. M., ReDfearN, J. W. T. (1956). An analysis of the frequencies of finger tremor in healthy subjects. Journal of Physiology (London), 134, 600-611.

Hays, W. L. (1981). Statistics (3rd ed.). New York: CBS College Publishing.
HEDGES, L. V., \& OLKIN, I. (1985). Statistical methods for meta-analysis. Orlando, FL: Academic Press.

IAIzzo, P. O., \& Pozos, R. S. (1982). Exercise-induced amplification modification of physiological action tremor of the ankle. Journal of Applied Physiology, 53, 1164-1170.

JoYCE, G. C., \& RACK, P. M. H. (1974). The effects of load and force on tremor at the normal human elbow joint. Journal of Physiology, 240, 375-396.

KoNDRASKE, G. V. (1986). A noncontacting human tremor sensor and measurement system. IEEE Transactions on Instrumentation \& Measurement, IM-35, 201-206.

KROEMER, K. H. E., \& MARRAS, W. S. (1981). Evaluation of maximal and submaximal static muscle exertions. Human Factors, 23, 643-653.

LIPPOLD, O. C. J. (1970). Oscillation in the stretch reflex arc and the origin of the rhythmical, 8-12 c/s component of physiological tremor. Journal of Physiology, 206, 359-382.

LIPPOLD, O. C. J., ReDFEARN, J. W. T., \& VuCo, J. (1960). The electromyography of fatigue. Ergonomics, 3, 120-131.

MARSDEN, C. D. (1978). The mechanisms of physiological tremor and their significance for pathological tremors. In J. E. Desmedt (Ed.), Physiological tremor, pathological tremor and clonus: Progress in clinical neurophysiology (Vol. 5, pp. 1-16). Basel: Karger.

Marshall, J., \& Walsh, G. (1956). Physiological tremor. Journal of Neurology, Neurosurgery, \& Psychiatry, 19, 260-267.

Matthews, P. B. C., \& Muir, R. B. (1980). Comparison of electromyogram spectra with force spectra during human elbow tremor. Journal of Physiology, 302, 427-441.

PUTZ-ANDERSON, V. (1988). The impact of automation on musculoskeletal disorders. In W. Karwowski, H. R. Parsaei, \& M. R. Wilhelm (Eds.), Ergonomics of hybrid automated systems I (pp. 645-651). Amsterdam: Elsevier.

RIETZ, R. R., \& STILEs, R. N. (1974). A viscoelastic-mass mechanism as a basis for normal postural tremor. Journal of Applied Physiology, 37, 852-860.

RoBson, J. G. (1962). An analysis of a human stretch reflex. Unpublished doctoral dissertation, Cambridge University.

Rosa, R. R., \& Colligan, M. J. (1988). Long workdays versus restdays: Assessing fatigue and alertness with a portable performance battery. Human Factors, 30, 305-317.

Rosa, R. R., Colligan, M. J., \& Lews, P. (1989). Extended workdays: Effects of 8-hour and 12-hour rotating shift schedules on performance, subjective alertness, sleep patterns, and psychosocial variables. Work \& Stress, 3, 21-32.

Rosa, R. R., Wheeler, D. D., Warm, J. S., \& Colligan, M. J. (1985). Extended workdays: Effects on performance and ratings of fatigue and alertness. Behavior Research Methods, Instruments, \& Computers, 17, 6-15.

Silverstein, B. A., Fine, L. J., \& ARmstrong, T. J. (1986). Hand wrist cumulative trauma disorders in industry. British Journal of Industrial Medicine, 43, 779-784.

STILES, R. N. (1976). Frequency and displacement amplitude relations for normal hand tremor. Journal of Applied Physiology, 40, 44-54.

StILES, R. N., \& RIETZ, R. R. (1977). Involuntary ankle oscillations from normal subjects. American Journal of Physiology, 233, 9-14.

Young, R. R. (1984). Physiological and enhanced physiological tremor. In L. J. Findley \& R. Capildeo (Eds.), Movement disorders: Tremor (pp. 127-134). New York: Oxford University Press.

Young, R. R., HAGBARTh, K. E. (1980). Physiological tremor enhanced by manoeuvres affecting the segmental stretch reflex. Journal of Neurology, Neurosurgery, \& Psychiatry, 43, 248-256.

(Manuscript received July 23, 1990; revision accepted for publication October 30, 1990.) 